\title{
Painéis de madeira aglomerada de Eucalyptus badjensis e Pinus spp.
}

\author{
Particleboards of Eucalyptus badjensis and Pinus spp.
}

\author{
Giuliano Ferreira Pereira1, Polliana D'Angelo Rios ${ }^{2}$, Rodrigo Buss ${ }^{3}$, Helena Cristina Vieira ${ }^{1}$, \\ Willian Grubert ${ }^{3}$, Alexsandro Bayestorff da Cunha ${ }^{2}$ e Ugo Leandro Belini ${ }^{4}$
}

\begin{abstract}
Resumo
No presente trabalho objetivou-se avaliar o potencial de utilização da madeira de Eucalyptus badjensis como alternativa para a produção de painéis de madeira aglomerada, bem como analisar o efeito da sua utilização em mistura com a madeira de Pinus spp. Nesse sentido, foram confeccionados painéis utilizando madeira de E. badjensis e de Pinus spp., nas composições homogênea e em camadas, utilizando $12 \%$ do adesivo ureia-formaldeído. Foram produzidos painéis com densidade nominal de $650 \mathrm{~kg}^{-3} \mathrm{~m}^{-3}$, dimensões de $40 \times 40 \times 1,55 \mathrm{~cm}$ (largura, comprimento e espessura), ciclo de prensagem utilizando pressão de 40 $\mathrm{kgf.cm}{ }^{-2}$ e temperatura de $180^{\circ} \mathrm{C}$ durante 8 minutos. Ademais, foi realizada a análise química da madeira em que foram determinados os teores de extrativos totais, cinzas, lignina e holocelulose. Além disso, foram executadas análises da geometria das partículas, sendo estudados o índice de esbeltez, razão de planicidade e área superficial específica. Para os painéis, as propriedades físicas e mecânicas analisadas foram: densidade aparente, absorção de água e inchamento em espessura após 2 e 24 horas, módulos de elasticidade e ruptura à flexão estática (MOE) e (MOR) e tração perpendicular. Com o presente estudo pode-se concluir que a madeira de E. badjensis, bem como suas misturas com Pinus spp. apresentam potencial para produção de painéis de madeira aglomerada.
\end{abstract}

Palavras-chave: Painéis de Madeira, Espécie não convencional, Mistura de espécies.

\begin{abstract}
In the present study we aimed to evaluate the potential use of Eucalyptus badjensis as an alternative for the production of particleboard, as well as to analyze the effect of its use in combination with the wood of Pinus spp. In this sense, were made particleboards using E. badjensis and Pinus spp., in homogeneous compositions and layered, using $12 \%$ urea-formaldehyde adhesive. Panels were produced with nominal density of $650 \mathrm{~kg} \cdot \mathrm{m}^{-3}$, dimensions of $40 \times 40 \times 1.55 \mathrm{~cm}$ (width, length and thickness), pressing cycle using $40 \mathrm{kgf.cm}{ }^{-2}$ pressure and temperature of $180^{\circ} \mathrm{C}$ for 8 minutes. Furthermore, through wood chemical analyses the total content of extractives, ash, lignin and holocelulose was determined. In addition, analyses were performed of the particle geometry and the slenderness ratio, flatness ratio and specific surface area was studied. For the panels, the physical and mechanical properties studied were: apparent density, water absorption and thickness swelling after 2 and 24 hours, modulus of elasticity (MOE), modulus of rupture (MOR). With this study it can be concluded that the wood of E. badjensis and its mixtures with Pinus spp. present potential for the production of particleboard.
\end{abstract}

Keywords: Wood Panels, Unconventional species, Mix of species.

\section{INTRODUÇÃO}

A espécie é uma das principais variáveis do processo produtivo de painéis de madeira aglomerada, uma vez que interage com todas as outras variáveis podendo exercer influências positivas ou negativas no produto final (MALONEY, 1993; IWAKIRI et al., 2004). Diferentes espécies produzem distintos tipos de matéria prima gerando desse modo diferentes tipos de partículas após o processamento (MALONEY, 1993).

\footnotetext{
${ }^{1}$ Mestre em Engenharia Florestal. UDESC - Universidade do Estado de Santa Catarina / Centro Agroveterinário. A. Luiz de Camões, 2090 - Dinheiro - 88520-000 - Lages, SC, Brasil. E-mail: giuliano.giiu@gmail.com; lenacristi@hotmail.com 2Professor Titular do Departamento de Engenharia Florestal. UDESC - Universidade do Estado de Santa Catarina / Centro Agroveterinário. Av. Luiz de Camões, 2090 - Dinheiro - 88520-000 - Lages, SC, Brasil. E-mail: polliana.rios@gmail.com; alexsandro.cunha@udesc.com

${ }^{3}$ Mestrando em Engenharia Florestal. UDESC - Universidade do Estado de Santa Catarina / Centro Agroveterinário. Av. Luiz de Camões, 2090 - Dinheiro - 88520-000 - Lages, SC, Brasil. E-mail: rodrigo.buss@gmail.com; w.grubert@live.com ${ }^{4}$ Professor Titular do Departamento de Desenho Industrial. UTFPR - Universidade Tecnológica Federal do Paraná. Campus Curitiba. Av. Sete de Setembro - Rebouças - 80230901 - Curitiba, PR, Brasil. E-mail: ubelini@yahoo.com.br
}

Sci. For., Piracicaba, v. 45, n. 114, p. 373-382, jun. 2017 DOI: dx.doi.org/10.18671/scifor.v45n114.13 
De acordo com Maloney (1993), para a produção de painéis reconstituídos as espécies mais recomendadas são aquelas que apresentam densidade de até $550 \mathrm{~kg} \cdot \mathrm{m}^{-3}$. A utilização de madeiras de baixa densidade resulta em alta razão de compactação dos painéis e maior área de contato entre as partículas, resultando assim em melhores propriedades de flexão estática e tração perpendicular (KELLY, 1997).

Por apresentarem propriedades desejáveis, concomitantemente com crescimento rápido e boa oferta de material no país, as principais espécies utilizadas como matéria-prima para produção de painéis de madeira aglomerada no Brasil são as dos gêneros Pinus e Eucalyptus (IWAKIRI et al., 2005a).

Dentre as espécies do gênero Eucalyptus, uma espécie que apresenta elevado potencial para a introdução em povoamentos comerciais no sul do Brasil, sobretudo no Planalto Catarinense e na região Centro-Sul do Paraná, é o Eucalyptus badjensis.

O Eucalyptus badjensis é proveniente da Austrália e apresenta como diferencial a elevada resistência às geadas. De acordo com Higa et al. (2002), mesmo com a ocorrência de geadas severas, são raros os danos aparentes nas mudas, como queima de folhas jovens e brotos.

Sobre a utilização tecnológica da espécie Mantero et al. (2008) concluíram que a espécie apresenta características anatômicas adequadas para a produção de papel, conferindo ao material, boa ligação entre as fibras, boa formação da folha, alta resistência à tração perpendicular e a ruptura, além de boa opacidade.

O Eucalyptus badjensis também apresenta potencial para extração de óleos essenciais. Antônio (2011) estudou a presença de óleos essenciais na espécie e concluiu que as folhas secas da espécie apresentam um bom rendimento, com alto teor de 1,8-cineol $(81,17 \%)$, um dos mais procurados metabólitos em óleos essenciais de Eucalyptus. Contudo, não foram encontrados na literatura estudos sobre a utilização da espécie para a produção de painéis de madeira.

Nesse contexto, estudos que avaliem o potencial da utilização da madeira de Eucalyptus badjensis, na confecção de painéis, são altamente relevantes. O presente estudo tem como objetivo estudar o potencial de utilização da espécie Eucalyptus badjensis na produção de painéis de madeira aglomerada, bem como estudar o efeito da sua utilização em diferentes misturas com a madeira de Pinus spp.

\section{MATERIAL E MÉTODOS}

\section{Coleta e preparo do material}

Para a confecção dos painéis utilizou-se madeira de E. badjensis e Pinus spp. A madeira de Eucalytpus badjensis foi fornecida por uma empresa de base florestal, sendo proveniente de um povoamento com 6 anos de idade. As árvores foram derrubadas e seccionadas em toras de 1,0 m, as quais foram transformadas em cavacos e posteriormente reduzidas aleatoriamente em partículas, por meio de um picador de martelo.

Por sua vez, a madeira de Pinus spp, por ser proveniente do processo produtivo de uma empresa de painéis reconstituídos, já se encontrava transformada em partículas no momento de sua chegada ao laboratório. A principal matéria-prima utilizada pela empresa consiste na madeira das espécies Pinus taeda e Pinus elliottii, sendo que no processo de produção, a empresa utiliza um mix dessas espécies, por esta razão foi denominada no presente trabalho como Pinus spp.

\section{Análise química}

O material utilizado nas análises foi padronizado com a utilização de peneiras de 40/60 Mesh, sendo selecionadas as partículas que passaram pela peneira de 40 Mesh e ficaram retidas na de 60 Mesh. Após a padronização dimensional, as amostras foram mantidas em sala de climatização, com umidade relativa de $60 \pm 5 \%$ e temperatura de $20 \pm 3{ }^{\circ} \mathrm{C}$. Determinou-se a porcentagem de extrativos totais NBR, 14660 (ABNT, 2004), teor de lignina TAPPI T413 (TAPPI, 1993), teor de cinzas TAPPI T222 (TAPPI, 2002). Por sua vez o teor de holocelulose foi determinado por diferença dos demais componentes (Holocelulose $=100-($ Extrativos totais + Teor de cinzas + Teor de lignina $)$ ).

\section{Densidade básica da madeira e Razão de compactação}

A densidade básica da madeira de E. badjensis foi calculada conforme os procedimentos descritos pela norma NBR 7190 (ABNT, 1997). Por sua vez para Pinus spp., a densidade básica foi calculada com base na média dos valores obtidos na literatura para a madeira da espécie. 
A razão de compactação dos painéis foi calculada por meio da equação 1.

Em que:

$$
R c=\frac{\text { dpainel }}{\text { dmadeira }}
$$

Rc = Razão de compactação;

Dpainel $=$ Densidade aparente do painel;

Dmadeira = Densidade básica da madeira.

Para painéis produzidos com misturas de espécies, foi utilizada a média das densidades das espécies para a realização do cálculo.

\section{Geometria das partículas}

O cálculo das variáveis pertinentes à geometria das partículas seguiu o modelo descrito por Trianoski (2010). Desse modo, foram mensuradas utilizando um paquímetro digital, a espessura, o comprimento e a largura de 500 partículas de cada espécie.

$\mathrm{O}$ índice de esbeltez foi calculado pela relação entre o comprimento e espessura das partículas e a razão de planicidade calculada pela relação entre a largura e espessura.

Por sua vez, o cálculo da área superficial foi realizado pelo método proposto por Moslemi (1974), por meio da equação 2:

Em que:

$$
\mathrm{AS}=\frac{2 *\left(\mathrm{E}^{*} \mathrm{~L}+\mathrm{L}^{*} \mathrm{C}+\mathrm{E}^{*} \mathrm{C}\right)}{\mathrm{E}^{*} \mathrm{~L}^{*} \mathrm{C}{ }^{*} \mathrm{Dm}}
$$

\section{(Equação 2)}

AS: área superficial das partículas $\left(\mathrm{cm}^{-2} / \mathrm{g}\right)$; E: Espessura média $(\mathrm{cm})$; L: Largura média $(\mathrm{cm}) ; \mathrm{C}$ : Comprimento médio $(\mathrm{cm})$; Dm: Densidade da madeira $\left(\mathrm{g} \cdot \mathrm{cm}^{-3}\right)$.

\section{Delineamento experimental}

Para a realização do experimento foi utilizado o delineamento inteiramente casualizado (DIC), constituído por 4 tratamentos, com 4 repetições (Tabela 1).

Tabela 1. Delineamento experimental dos painéis.

Table 1. Experimental design of the panels.

\begin{tabular}{llc}
\hline T & Espécie (s) & TA (\%) \\
\hline T1 & E. badjensis & 12 \\
T2 & Pinus spp. & 12 \\
T3 & Mistura homogênea & 12 \\
T4 & Mistura multicamada & 12 \\
\hline
\end{tabular}

Os painéis de mistura homogênea forma confeccionados com $50 \%$ de particulas de E. badjensis e 50\% de partículas de Pinus spp., por sua vez os painéis multicamada foram confeccionados na proporção 25\% Pinus spp./50\% E. badjensis/25\% Pinus spp.

\section{Produção dos painéis}

Primeiramente, as partículas foram secas em estufa a $60{ }^{\circ} \mathrm{C}$, até atingirem valores de $4 \%( \pm 2 \%)$ de umidade. O teor de umidade das partículas foi determinado por meio de uma termobalança de infravermelho. Com as partículas secas, iniciou-se a produção dos painéis, nas dimensões de $40 \mathrm{x}$ $40 \times 1,55 \mathrm{~cm}$ (largura, comprimento e espessura).

Foi utilizado $12 \%$ do adesivo ureia-formaldeido calculado com base no peso seco do material, o mesmo apresentava $66,4 \%$ de teor de sólidos. Para a aspersão do adesivo, foi utilizada uma encoladeira, do tipo tambor giratório dotada de um copo graduado e uma pistola a ar comprimido. No presente estudo não foi aplicada emulsão de parafina.

Posteriormente, o material passou por uma pré-prensagem em prensa hidráulica manual, utilizando $5 \mathrm{kgf} / \mathrm{cm}^{2}$ durante 5 minutos. Em seguida, o material passou por uma prensa hidráulica automática, utilizando temperatura de $180^{\circ} \mathrm{C}$, pressão de $40 \mathrm{kgf} / \mathrm{cm}^{2}$ e tempo de 8 minutos.

Após a prensagem, os painéis foram climatizados, de acordo com a norma da NBR 14660 (ABNT, 2004), com temperatura de $20 \pm 2{ }^{\circ} \mathrm{C}$ e umidade relativa de $65 \pm 5 \%$. 
Pereira et al. - Painéis de madeira aglomerada de Eucalyptus badjensis e Pinus spp.

\section{Testes físicos e mecânicos}

Para os testes físicos e mecânicos, as normas utilizadas estão representados na Tabela 2.

Tabela 2. Normas utilizadas na condução dos ensaios físicos e mecânicos.

Table 2. Standards used in conducting physical and mechanical tests.

\begin{tabular}{ll}
\hline Propriedade Avaliada & Norma \\
\hline $\begin{array}{l}\text { Densidade } \\
\text { Absorção de água 2 e 24 horas }\end{array}$ & ASTM D 1037 (ASTM, 2002) \\
Inchamento em espessura 2 e 24 horas & \\
\hline Tração perpendicular & DIN 52362 (DIN, 1982) \\
$\begin{array}{l}\text { Módulo de elasticidade à flexão estática } \\
\text { Módulo de ruptura à flexão estática }\end{array}$ & NBR 14810 (ABNT, 2006) \\
\hline $\begin{array}{l}\text { Arrancamento de parafuso } \\
\text { Teor de umidade }\end{array}$ & N
\end{tabular}

Os resultados obtidos foram comparados com a norma NBR 14810 (ABNT, 2006).

\section{Análise Estatística}

Primeiramente, com o intuito de verificar a normalidade e homogeneidade dos dados, foram realizados, respectivamente, os testes Shapiro-Wilk e Bartlett,(SHAPIRO; WILK, 1965). Em decorrência da falta de homogeneidade e normalidade dos dados de Inchamento em espessura, foi necessária a transformação matemática do tipo Box-Cox para a variável. Para os dados de geometria das partículas, mesmo após a utilização de transformações matemáticas, os dados não apresentaram homegeneidade e normalidade, desse modo, foi utilizado o teste não-paramétrico de Kruskall-Wallis. Em todos os outros casos, foi realizado o teste de médias de Skott-Knott, ao nível de 95\% de confiança.

\section{RESULTADOS E DISCUSSÃO}

\section{Caracterização química}

Os valores referentes à análise química quantitativa da madeira de E. badjensis e de Pinus spp. estão representados na Tabela 3

Tabela 3. Valores médios das propriedades químicas dos materiais.

Table 3. Mean values of the chemical properties of the materials.

\begin{tabular}{lcccc}
\hline Espécie & Holocelulose & Lignina & Extrativos & Cinzas \\
\hline \multirow{2}{*}{ E. badjensis } & $70,73 \mathrm{a}$ & $24,63 \mathrm{a}$ & $4,02 \mathrm{a}$ & $0,59 \mathrm{a}$ \\
& $(5,54)$ & $(5,56)$ & $(6,74)$ & $(10,54)$ \\
\hline \multirow{2}{*}{ Pinus spp. } & $64,92 \mathrm{~b}$ & $27,64 \mathrm{a}$ & $6,92 \mathrm{~b}$ & $0,62 \mathrm{a}$ \\
& $(0,68)$ & $(8,86)$ & $(2,62)$ & $(2,77)$ \\
\hline
\end{tabular}

Médias seguidas de letras diferentes não diferem entre si pelo teste de Skott-knott ao nível de confiança 95\%. CV (Coeficiente de variação entre parênteses).

O teor de lignina obtido para Pinus spp. não apresentou diferença estatística quando comparado ao E. badjensis. Contudo, de acordo com Andrade (2006), de maneira geral em coníferas há maior teor de lignina do que em folhosas, tal fato pode influenciar na colagem das partículas durante a produção dos painéis, uma vez que a lignina pode atuar como agente cimentante. Pelo fato do E. badjensis apresentar-se estatisticamente semelhante ao Pinus spp., pode-se afirmar que a espécie encontra-se adequada para a confecção de painéis de madeira aglomerada, no que tange essa variável.

Para teor de extrativos, o percentual obtido para Pinus spp. (6,02\%) foi superior estatisticamente ao encontrado para E. badjensis (4,03\%). Cloutier (1998) afirma que, espécies com baixos percentuais de extrativos são as mais recomendadas para a produção de painéis de madeira, uma vez que a presença de grande quantidade de extrativos na madeira pode ocasionar estouros nos painéis ao final da prensagem, além de provocar uma menor eficiência na colagem de partículas. 
Devido ao fato de apresentar teor de extrativos inferior à espécie mais utilizada atualmente no Brasil para produção de aglomerado, pode-se concluir que o E. badjensis encontra-se com percentuais adequados para a produção desse tipo de painel.

As madeiras de E. badjensis e de Pinus spp. não apresentaram diferença estatística para teor de cinzas. Os teores obtidos para os dois materiais podem ser considerados baixos, sendo desse modo desejáveis para a produção de painéis de madeira aglomerada, uma vez que madeiras de espécies com teores de cinza elevados podem promover o desgaste das ferramentas de corte utilizadas na conversão das toras em partículas.

\section{Densidade básica da madeira}

Os valores médios de densidade básica obtidos para as árvores de E. badjensis não diferiram estatisticamente entre si.

A densidade média obtida para o E. badjensis foi de $476 \mathrm{~kg} . \mathrm{m}^{-3}$, sendo semelhante a encontrada na literatura para outras espécies do gênero Eucalyptus, como a obtida por Nisgoski et al. (1998), para árvores de E. benthamii com 7 anos de idade $\left(470 \mathrm{~kg} \cdot \mathrm{m}^{-3}\right)$.

\section{Geometria das partículas}

Com a análise da tabela 4, é possível observar que as partículas de Pinus spp. apresentaram Índice de esbeltez superior aos obtidos para E. badjensis.

Tabela 4. Valores médios dos Índice de esbeltez, Razão de planicidade e Área superficial. Table 4. Mean slenderness index, flatness ratio and surface area.

\begin{tabular}{lccc}
\hline \multirow{2}{*}{ Espécie } & IE & Pla & AS \\
\cline { 2 - 4 } & & $\mathbf{( \mathbf { c m } ^ { - 2 } \mathbf { g } )}$ & \\
\hline \multirow{2}{*}{ E. badjensis } & $21,08 \mathrm{~b}$ & $7,09 \mathrm{~b}$ & $81,86 \mathrm{a}$ \\
& $(36,74)$ & $(34,83)$ & $(35,93)$ \\
\hline \multirow{2}{*}{ Pinus spp. } & $53,47 \mathrm{a}$ & $11,07 \mathrm{a}$ & $72,23 \mathrm{~b}$ \\
\hline
\end{tabular}

Médias seguidas de letras diferentes apresentam diferença significativa pelo teste de Kruskal-Wallis ao nível de $95 \%$ de confiança. IE: Índice de esbeltez, Pla: Razão de planicidade, AS: Área Superfícial. CV: Coeficiente de variação, entre parênteses.

De acordo com Alberto (1992), isso pode influenciar na área de contato entre as partículas, nas propriedades mecânicas e no consumo de adesivo, sendo que índices de esbeltez maiores são mais desejáveis, uma vez que em geral conferem aos painéis maior resistência mecânica e estabilidade dimensional.

Na literatura os resultados obtidos apresentam grande variação. Para partículas de Pinus spp., Iwakiri (1989) obteve Índice de esbeltez de 30,40, para Pinus taeda Trianoski (2010) obteve 10,78. Por sua vez Naumann et al. (2008), observaram 24,68 para E. urophylla. Trianoski (2010) atribui essa variação às diferenças nas características das madeiras das espécies, tipos de equipamento utilizados para obtenção dos cavacos, além da espessura de partícula desejada.

De forma análoga ao índice de esbeltez, para a variável razão de planicidade, as partículas de Pinus spp. apresentaram valores superiores aos obtidos para E. badjensis. De acordo com Alberto (1992), a razão de planicidade afeta volume relativo de ar do colchão, a área superficial específica e o consumo de adesivo, sendo que valores maiores são mais desejáveis, uma vez que esse aumento ocasiona uma melhora nas condições de distribuição de adesivo.

Para área superficial específica, a espécie E. badjensis apresentou os menores valores. Maloney (1993) e Moslemi (1974) afirmam que a quantidade de adesivo está estritamente associada à área superficial específica das partículas. Dessa forma, partículas com maior área superficial específica requerem quantidades maiores de adesivo em relação a partículas com área superficial específica menor.

\section{Propriedades físicas}

As médias obtidas para as propriedades físicas dos painéis estão apresentadas na tabela 5. 
Tabela 5. Médias das propriedades físicas dos painéis.

Table 5. Means of the physical properties of panels.

\begin{tabular}{|c|c|c|c|c|c|c|c|}
\hline & \multirow{2}{*}{$\begin{array}{c}\text { DA } \\
\left(\mathrm{kg} \cdot \mathrm{m}^{-3}\right)\end{array}$} & \multirow[t]{2}{*}{ RC } & \multirow[t]{2}{*}{ TU } & \multirow[t]{2}{*}{ AA2h } & \multirow{2}{*}{$\begin{array}{c}\text { AA24h } \\
(\%)\end{array}$} & \multirow[t]{2}{*}{ IE2h } & \multirow[t]{2}{*}{ IE24h } \\
\hline & & & & & & & \\
\hline E.badjensis & $\begin{array}{c}601 a \\
(5,48) \\
\end{array}$ & $\begin{array}{l}1,26 c \\
(4,39)\end{array}$ & $\begin{array}{l}9,42 a \\
(7,58)\end{array}$ & $\begin{array}{l}50,9 a \\
(8,60)\end{array}$ & $\begin{array}{l}69,3 a \\
(4,77)\end{array}$ & $\begin{array}{l}17,6 \mathrm{~b} \\
(7,52)\end{array}$ & $\begin{array}{l}20,2 a \\
(7,26)\end{array}$ \\
\hline Pinus spp & $\begin{array}{c}603 a \\
(4,58)\end{array}$ & $\begin{array}{l}1,66 a \\
(5,29)\end{array}$ & $\begin{array}{c}10,53 b \\
(6,74)\end{array}$ & $\begin{array}{c}94,9 c \\
(11,67)\end{array}$ & $\begin{array}{c}104,1 \mathrm{c} \\
(10,90)\end{array}$ & $\begin{array}{l}17,2 b \\
(6,97)\end{array}$ & $\begin{array}{c}19,2 a \\
(12,89) \\
\end{array}$ \\
\hline Mist. Hom & $\begin{array}{l}604 a \\
(6,06)\end{array}$ & $\begin{array}{l}1,44 \mathrm{~b} \\
(4,72)\end{array}$ & $\begin{array}{l}9,62 a \\
(7,06)\end{array}$ & $\begin{array}{l}67,3 b \\
(4,66) \\
\end{array}$ & $\begin{array}{l}82,7 b \\
(9,61) \\
\end{array}$ & $\begin{array}{l}16,1 \mathrm{a} \\
(4,82)\end{array}$ & $\begin{array}{l}22,9 \mathrm{~b} \\
(7,61)\end{array}$ \\
\hline Mist. Multi & $\begin{array}{c}607 a \\
(5,86)\end{array}$ & $\begin{array}{l}1,43 b \\
(5,80)\end{array}$ & $\begin{array}{c}10,57 \mathrm{~b} \\
(6,59)\end{array}$ & $\begin{array}{l}68,0 b \\
(8,21)\end{array}$ & $\begin{array}{c}85,3 b \\
(10,88)\end{array}$ & $\begin{array}{l}18,8 \mathrm{c} \\
(4,74)\end{array}$ & $\begin{array}{l}28,5 c \\
(9,78)\end{array}$ \\
\hline
\end{tabular}

Mist: Mistura; AA2h: Absorção de água em 2 horas; AA24h: Absorção de água em 24 horas; IE2h: Inchamento em espessura em 2 horas; IE24h: Inchamento em espessura em 24 horas. NOTA: Médias seguidas de letras diferentes não diferem entre si pelo teste de Skott-knott ao nível de confiança 95\%. CV (Coeficiente de variação entre parênteses).

\section{Densidade aparente}

Para densidade aparente dos painéis a análise estatística não indicou diferença significativa para as diferentes composições de painel (Tabela 5). As médias obtidas variaram de $601 \mathrm{~kg} \cdot \mathrm{m}^{-3}$ a 607 kg.m-3 ${ }^{3}$ desse modo os painéis podem ser classificados como de média densidade de acordo a NBR 14810 (ABNT, 2006).

As densidades dos painéis obtidas no presente estudo foram inferiores a densidade planejada $\left(650 \mathrm{~kg} \cdot \mathrm{m}^{-3}\right)$. Esse fato ocorreu em outros estudos na literatura, como o de Rios et al. (2016) para painéis de Pinus patula e Pinus spp que obtiveram valores médios de $565 \mathrm{~kg} . \mathrm{m}^{-3}$ para painéis com densidade nominal de $650 \mathrm{~kg} \cdot \mathrm{m}^{-3}$.

Iwakiri et al. (2012) e Santos et al. (2009) atribuem a diferença entre a densidade obtida e a densidade nominal às condições operacionais em nível laboratorial, uma vez que nesse ambiente não é possível reproduzir a automação e precisão do processo industrial, podendo acarretar entre outros, a perda de partículas durante o manuseio nas fases anteriores à consolidação do painel na prensa quente.

Outro fator que contribuiu para a diferença obtida foi o deslizamento lateral de partículas, fenômeno que ocasiona um aumento nas dimensões dos painéis após a prensagem.

\section{Razão de compactação}

Para a variável razão de compactação foi observada diferença estatística. As médias variaram de 1,26 (E.badjensis) a 1,66 (Pinus spp.). Segundo Maloney (1993), a faixa ideal para a razão de compactação é de 1,3 a 1,6, sendo que painéis com valores de razão de compactação superiores a 1,6 apresentam menores valores para propriedades físicas e maiores nas propriedades mecânicas, em contrapartida, painéis com valores, inferiores a 1,3, apresentam redução nas propriedades mecânicas.

Os painéis homogêneos de E. badjensis apresentaram média inferior a desejável, em contrapartida os painéis homogêneos de Pinus spp. apresentaram média superior a desejável. Por sua vez, os painéis produzidos, com misturas das duas espécies, enquadraram-se na faixa ideal para a propriedade.

\section{Teor de umidade}

Para teor de umidade as médias variaram de $9,42 \%$ a 10,57\%, desse modo os painéis de todas as composições atenderam aos requisitos estipulados pela norma NBR 14810 (ABNT, 2006), que estabelece valores de teor de umidade entre $5 \%$ e $11 \%$.

A umidade de equilíbrio obtida foi inferior a da sala de climatização (12\%). Esta diminuição da higroscopicidade em painéis reconstituídos é explicada pela redução da madeira em partículas e posterior incorporação de adesivo, parafina e outros aditivos, além da aplicação de altas temperaturas e pressão durante a consolidação do painel, as quais promovem a perda ou rearranjo das regiões higroscópicas da madeira, deixando o painel menos reativo a água (MENDES, 2001).

\section{Absorção de água e inchamento em espesura}

Para Absorção de água pode-se verificar que os painéis de Pinus spp. apresentaram maiores médias, seguido das misturas e dos painéis homogêneos de E. badjensis. que foram estatisticamente 
semelhantes, tanto para 2 horas quanto para 24 horas de imersão em água. Esse fato pode ser correlacionado com razão de compactação, uma vez que os painéis de Pinus spp. apresentaram maiores valores para a variável. Segundo Iwakiri et al. (2005b) painéis com elevada razão de compactação apresentam maior liberação das tensões de compressão impostas durante a prensagem e por conseguinte maior absorção de água.

Para inchamento em espessura, pode-se observar que os painéis puros de E. badjensis e Pinus spp. foram semelhantes entre si, tanto para 2 quanto para 24 horas de imersão em água. Este resultado demonstra que a madeira de E. badjensis possui caraterísticas adequadas para essa propriedade, uma vez que apresentou resultados semelhantes ou superiores aos obtidos para madeira de uma espécie amplamente utilizada na produção de painéis de madeira aglomerada.

Em relação à norma NBR 14810 (ABNT, 2006) nenhum dos tratamentos atingiu os valores mínimos estipulados para o ensaio de Inchamento em espessura em 2 horas (8\%). Um fator que contribuiu para os elevados valores obtidos foi a não utilização de emulsão de parafina, tendo em vista que tendo em vista que sua utilização diminui significativamente a higroscospicidade do material. Segundo Guimarães Júnior et al. (2013) a adição de 1\% de parafina na produção de painéis de madeira aglomerada ocasiona uma redução de aproximadamente $11 \%$ no inchamento em espessura.

\section{Propriedades mecânicas}

As médias obtidas para as propriedades mecânicas dos painéis estão apresentadas na tabela 6 .

Tabela 6. Valores médios das propriedades mecânicas dos painéis.

Table 6. Means of mechanical properties of panels.

\begin{tabular}{lccccc}
\hline & $\begin{array}{c}\text { TP } \\
\text { (MPa) }\end{array}$ & $\begin{array}{c}\text { MOE } \\
\text { (MPa) }\end{array}$ & $\begin{array}{c}\text { MOR } \\
\text { (MPa) }\end{array}$ & $\begin{array}{c}\text { APT } \\
\text { (N) }\end{array}$ & $\begin{array}{c}\text { APS } \\
\text { (N) }\end{array}$ \\
\hline \multirow{2}{*}{ E. badjensis } & $0,58 \mathrm{a}$ & $1282,90 \mathrm{c}$ & $15,80 \mathrm{c}$ & $1523,93 \mathrm{~b}$ & $1642,5 \mathrm{a}$ \\
& $(5,14)$ & $(22,39)$ & $(23,08)$ & $(21,66)$ & $(25,67)$ \\
\hline \multirow{2}{*}{ Pinus spp. } & $0,28 \mathrm{~b}$ & $1139,72 \mathrm{~d}$ & $14,70 \mathrm{c}$ & $755,93 \mathrm{~d}$ & $1081,5 \mathrm{c}$ \\
& $(10,35)$ & $(34,64)$ & $(30,50)$ & $(26,15)$ & $(23,97)$ \\
\hline \multirow{2}{*}{ Mist. Hom } & $0,60 \mathrm{a}$ & $1593,54 \mathrm{~b}$ & $20,82 \mathrm{~b}$ & $1716,93 \mathrm{a}$ & $1580,06 \mathrm{a}$ \\
& $(13,58)$ & $(24,11)$ & $(7,06)$ & $(11,56)$ & $(21,38)$ \\
\hline \multirow{2}{*}{ Mist. Multi } & $0,51 \mathrm{a}$ & $1895,17 \mathrm{a}$ & $25,31 \mathrm{a}$ & $1385,87 \mathrm{c}$ & $1385,63 \mathrm{~b}$ \\
& $(13,75)$ & $(17,48)$ & $(6,59)$ & $(22,03)$ & $(27,78)$ \\
\hline
\end{tabular}

MOE: Módulo de elasticidade, MOR: Módulo de Ruptura, APT: Arrancamento de parafuso (Topo), APS: Arrancamento de parafuso (Superfície), TP: Tração perpendicular. NOTA: Médias seguidas de letras diferentes não diferem entre si pelo teste de Skott-knott ao nível de confiança $95 \%$. CV (Coeficiente de variação entre parênteses).

Para tração perpendicular as composições homogêneas de E. badjensis e as misturas homogênea e multicamada apresentaram médias estatisticamente superiores a média obtida para Pinus spp.. Este resultado pode ser explicado pelo maior índice de esbeltez das partículas de Pinus spp. De acordo com Carvalho et al. (2014), partículas com maiores índices de esbeltez apresentam uma menor disponibilidade de resina, fato que ocasiona uma colagem menos eficiente das partículas e por conseguinte redução dos valores de tração perpendicular.

Em relação à norma NBR 14810 (ABNT, 2006), apenas os painéis confeccionados com Pinus spp. não atenderam aos requisitos da norma, indicando que a madeira de E. badjensis apresenta propriedades adequadas no que tange essa variável.

Para módulo de elasticidade e ruptura a flexão estática, todas as composições analisadas diferiram estatisticamente entre si, sendo que as maiores médias foram obtidas para mistura multicamada. Segundo Sanches (2012), isso pode ser explicado pelo gradiente de densidade que se forma ao longo da espessura do painel, ocasionando maior densificação das camadas superficiais e desse modo maiores valores para MOE e MOR.

A norma NBR 14810 (ABNT, 2006) não estabelece valores para MOE. Por sua vez, para MOR as composições puras de Pinus spp. e E. badjensis não atenderam aos valores estabelecidos pela NBR 14810 (ABNT, 2006). O fato de alguns tratamentos não atingirem os valores estipulados para a variável pode ser atribuído densidade dos painéis (601 kg.m- ${ }^{3}$ a $\left.607 \mathrm{~kg} \cdot \mathrm{m}^{-3}\right)$ não ter atingido a densidade desejada (650 kg.m-3 ${ }^{3}$. Segundo Mendes (2010) a cada incremento de $10 \mathrm{~kg} \cdot \mathrm{m}^{-3}$ na densidade dos painéis ocorre um aumento no MOR de aproximadamente 1,30 MPa. 
Pereira et al. - Painéis de madeira aglomerada de Eucalyptus badjensis e Pinus spp.

Para o ensaio arrancamento de parafuso, tanto para topo quanto para superfície, os painéis produzidos utilizando exclusivamente a madeira de Pinus spp. apresentaram os menores valores, fato que indica a influência positiva da utilização da madeira de E. badjensis, bem como suas misturas no que tange essa variável.

Em relação à NBR 14810 (ABNT, 2006), todas as composições enquadraram-se nos padrões de qualidade estipulados para o ensaio de superfície (1000N) e apenas os painéis homogêneos de Pinus spp. $(755,93 \mathrm{~N})$, não atenderam aos requisitos da norma para o ensaio de topo (800N). Este fato pode ser explicado pelo alto Índice de esbeltez das partículas de Pinus spp.. De acordo com Vital et al. (1992), partículas com menores índices de esbeltez apresentam valores mais elevados para tração perpendicular, pelo fato de apresentarem uma maior disponibilidade de resina e desse modo uma colagem mais eficiente.

\section{CONCLUSÕES}

A espécie E. badjensis apresenta propriedades químicas adequadas para a produção de painéis de madeira aglomerada; uma vez que apresentou resultados satisfatórios para todas as análises realizadas.

Os painéis do presente estudo podem ser classificados como de média densidade de acordo com a NBR 14810 (ABNT, 2006).

Para teor de umidade todas as composições enquadraram-se na faixa recomendada pela NBR 14810 (ABNT, 2006).

Para razão de compactação a mistura das madeiras de E. badjensis e Pinus spp. apresentou efeito positivo uma vez que os painéis produzidos com misturas das duas espécies enquadraram-se na faixa ideal para a propriedade.

A madeira de E. badjensis, bem como sua utilização em misturas com Pinus spp. apresentam potencial de utilização no processo produtivo de painéis de madeira, uma vez que apresentaram valores estatisticamente superiores ou semelhantes a madeira de Pinus spp. para a maior parte das propriedades físicas e mecânicas analisadas no presente estudo. Contudo para que sejam atingidos os valores recomendados pelas normas de comercialização são necessários ajustes no processo produtivo dos painéis, principalmente em relação à densidade e aplicação de redutores de higroscopicidade.

\section{AGRADECIMENTOS}

A FAPESC e a SED/SC por meio do programa UNIEDU pelo auxílio financeiro e institucional e as empresas Klabin e Bonet pelo fornecimento da matéria prima utilizada para a confecção dos painéis.

\section{REFERÊNCIAS BIBLIOGRÁFICAS}

ABNT - ASSOCIAÇÃO BRASILEIRA DE NORMAS TÉCNICAS. NBR 14810: Chapas de madeira aglomerada. Rio de Janeiro, 2006. 51 p.

ABNT - ASSOCIAÇÃO BRASILEIRA DE NORMAS TÉCNICAS. NBR 14660: Madeira - Amostragem e preparação para análise. Rio de Janeiro, 2004.

ABNT - ASSOCIAÇÃO BRASILEIRA DE NORMAS TÉCNICAS. NBR 7190: Projeto de estruturas de madeira. Rio de Janeiro, 1997. 107 p.

ALBERTO, M. M. M. Efeito da geometria das partículas e da densidade sobre as propriedades de painéis estruturais "waferboards". 1992. 122 p. Dissertação (Mestrado em Ciências Florestais) - Universidade Federal do Paraná, Curitiba, 1992.

ANDRADE, A. S. Qualidade da madeira, celulose e papel em Pinus taeda L.: influência da idade e classe de produtividade. 94 p. Dissertação (Mestrado em Engenharia Florestal) - Universidade Federal do Paraná, Curitiba, 2006. 
ANTÔNIO, R. D. Caracterização fitoquímica, morfoanatomia e atividades biológicas de Eucalyptus badjensis Beuzev e Welch, Myrtaceae. 2011. 110 p. Dissertação (Mestrado em Ciências farmacêuticas) Universidade Federal do Paraná, Curitiba, 2011.

ASTM - AMERICAN SOCIETY FOR TESTING ABD MATERIALS. Annual book of ASTM: D-1037 - Standard methods of evaluating properties of wood-base fiber and particles materials. Philladelphia, 2002.

CARVALHO, A. G.; ZANUNCIO, A. J. V.; MENDES, R. F; MORI, F. A.; SILVA, M. G.; MENDES, L. M. Adesivos tânicos de Stryphnodendron adstringens (Mart.) Coville na produção de painéis aglomerados. Revista Árvore, Viçosa, v. 38, n. 1, p. 195-202, 2014.

CLOUTIER, A. Oriented strandboard (OSB): raw material, manufacturing process, properties and uses, In: SEMINÁRIO INTERNACIONAL SOBRE PRODUTOS SÓLIDOS DE MADEIRA DE ALTA TECNOLOGIA. 1., 1998, Belo Horizonte. Anais... Belo Horizonte: Viçosa, 1998. p. 173-185.

DIN - NORMEN FÜR HOLZFASERPLATEN SPANPLATTEN SPERRHOLZ. Testing of wood chipboards bending test, determination of bending strength: DIN 52362. Berlin, 1982. 2 p.

GUIMARÃES JÚNIOR, J. B.; ARAÚJO, B. L. M.; LOPES, O. P.; MENDES, R. F.; MENDES, L. M. Produção de painéis aglomerados da madeira de desrama de Acacia mangium. Pesquisa Florestal Brasileira, Brasília, v.33, n.76, p. 387-391, 2013.

HIGA, R. C. V.; HIGA, A. R.; ALVES, E. C. Comportamento de progênies de Eucalyptus badjensis Beuzev. \& Welch em dois locais da Região Sul do Brasil. Boletim de Pesquisa Florestal, Colombo, n. 45, p. 89-97, 2002.

KELLY, M. W. Critical literature review of relationship between processing parameters and physical properties of particleboard. Raleigh: USDA, 1977. 70 p. (Forest Service Report, FPI 10).

IWAKIRI, S. A influência das variáveis de processamento sobre propriedades das chapas de partículas de diferentes espécies de Pinus. 130 p. Tese (Doutorado em Ciências Florestais) - Setor de Ciências Agrárias, Universidade Federal do Paraná, Curitiba, 1989.

IWAKIRI, S.; MATOS, J. L. M. DE; TRIANOSKI, R.; PRATA, J. G. Produção de painéis aglomerados homogêneos e multicamadas de Melia azedarach (CINAMOMO) e Pinus taeda com diferentes teores de resina. Cerne, Lavras, v. 18,n. 3, p. 465-470, 2012.

IWAKIRI, S. KEINERT JÚNIOR, S; MENDES, L. M; ALBUQUERQUE, C. E. D; LATORRACA, J. E. F. Painéis de Madeira. Curitiba: FUPEF, 2005a. 196 p.

IWAKIRI, S. Produção de painéis aglomerados de alta densificação com uso de resina melamina-uréiaformaldeído. Cerne, Lavras, v. 11, n. 4, p. 323-328, 2005b.

IWAKIRI, S.; SHIMIZU, J.; SILVA, J. C.; DEL MENEZZI, C. H. S.; PUERINGHER, C. A.; VENSON, I.; LARROCA, C. Produção de painéis de madeira aglomerada de Grevillea Robusta A. Cunn ex R. Br. Revista Árvore, Viçosa, v. 28, n. 6, p. $883-887,2004$.

LONGO, B. L.; CUNHA, A. B.; RIOS, P. D.; TEREZO, R. F; ALMEIDA, C. C. F. Caracterização tecnológica de painéis particulados produzidos com resíduos de cinco espécies tropicais comerciais. Scientia Forestalis, Piracicaba. v. 43, n. 108. p. 907-917, 2015.

MALONEY, T. M. Modern particleboard and dry-process fiberboard manufacturing. 2. ed. São Francisco: Miller Freeman, 1993. 689 p. 
Pereira et al. - Painéis de madeira aglomerada de Eucalyptus badjensis e Pinus spp.

MANTERO, C.; FERNÁNDEZ, V.; ROZ, A. Anatomía, peso específico aparente de la madera y porcentaje de corteza en orígenes de dos especies de Eucalyptus cultivadas em Uruguay (Eucalyptus dunnii Maiden y Eucalyptus badjensis Beuzev.\& Welch). Agrociencia. Montevidéu. v.22, n.1, p 20-30, 2008.

MENDES, L. M. Pinus spp.na produção de painéis de partículas orientadas. Curitiba, 2001. 156 p. Tese (Doutorado em Engenharia Florestal) - Universidade Federal do Paraná, Curitiba, 2001.

MENDES, R. F.; MENDES, L. M.; ABRANCHES, R. A. S.; SANTOS, R. C.; GUIMARÃES JÚNIOR, J. B.; Painéis aglomerados produzidos com bagaço de cana em associação com a madeira de eucalipto. Scientia Forestalis, Piracicaba, v. 38, n. 86, p. 285-295, 2010.

MOSLEMI, A. A. Particleboard. Illionis: Southern Illionis University, 1974. v. 2, 245 p.

NAUMANN, R. B.; VITAL, B. R.; CARNEIRO, A. de. C. O.; DELLA LUCIA, R. M.; SILVA, J. de C.; CARVALHO, A. M. M. L.; COLLI, A. Propriedades de chapas fabricadas com partículas de madeira de Eucalyptus urophylla S. T. Blake e de Schizolobium amazonicum Herb. Revista Árvore, Viçosa, v. 32, n. 6, p. 1143-1150, 2008.

NISGOSKI, S.; MUNIZ, G. I. B.; KLOCK, U. Caracterização anatômica da madeira de Eucalyptus benthamii Mainden et Cambage. Ciência Florestal, Santa Maria, v.8, n.1, p. 67-76, 1998.

RIOS, P. D. A.; PEREIRA, G. F.; VIEIRA, H. C.; GRUBERT, W.; CUNHA, A. B.; BRAND, M. Avaliação do potencial da madeira de Pinus patula Schltdl. \& Cham para a produção de painéis de madeira aglomerada. Scientia Forestalis, Piracicaba. v. 44. n. 110, 2016.

SANCHES, L. F. Qualidade de painéis aglomerados produzidos com mistura de madeiras de quatro espécies florestais. 2012. 80p. Dissertação (Mestrado em Ciências florestais) - Universidade Estadual do Centro-Oeste. Irati, 2012.

SANTOS, R. C.; MENDES, L. M.; MORI, F. A.; MENDES, R. F. Chapas de partículas aglomeradas produzidas a partir de resíduos gerados após a extração do óleo da madeira de candeia (Eremanthus erythropappus). Scientia Forestalis, Piracicaba, v. 37, n. 84, p. 437-446, 2009.

SHAPIRO, S. S.; WILK, M. B. An analysis of variance test for normality (complete samples). Biometrika, Cambridge, v. 52, n. 3/4, p. 591-611, 1965.

TAPPI - TECHNICAL ASSOCIATION OF THE PULP AND PAPER INDUSTRY. TAPPI T222: Acid-insoluble lignin in wood and pulp, Test Method. Atlanta, 2002. 5 p.

TAPPI - TECHNICAL ASSOCIATION OF THE PULP AND PAPER INDUSTRY TAPPI 413: Ash in wood, pulp, paper and paperboard: combustion at $900^{\circ} \mathrm{C}$, Test Method. Atlanta, 1993. 4 p.

TRIANOSKI, R., Avaliação do potencial de espécies florestais alternativas de rápido crescimento para produção de painéis de madeira aglomerada. Curitiba, 2010. 260 p. Dissertação (Mestrado em Engenharia Florestal) - Setor de Ciências Agrárias, Universidade Federal do Paraná. 2010.

VITAL, B. R.; HASELEIN, C. R.; DELLA LUCIA, R. M. Efeito da geometria das partículas nas propriedades das chapas de madeira aglomerada de Eucalyptus grandis (Hill ex-Maiden). Revista Árvore, Viçosa. v.16, n. 1, p. 88-96, 1992.

Recebido em 27/04/2016

Aceito para publicação em 06/03/2017 